\title{
OVERALL SPLENECTOMY RATES STABLE DESPITE INCREASING USAGE OF ANGIOGRAPHY IN THE MANAGEMENT OF HIGH GRADE BLUNT SPLENIC INJURY
}

\author{
AUTHORS: \\ Scott C. Dolejs MD ${ }^{1}$, Surgery Resident, sdolejs@iupui.edu \\ Stephanie A. Savage, MD MS ${ }^{1}$, Associate Professor of Surgery, stasava@iupui.edu \\ Jennifer L. Hartwell, MD ${ }^{1}$, Assistant Professor of Surgery, jlhartwe@iu.edu \\ Ben L. Zarzaur MD MPH ${ }^{1}$, Associate Professor of Surgery, bzarzaur@iupui.edu \\ AFFILIATIONS: ${ }^{1}$ Indiana University School of Medicine, Department of Surgery \\ CORRESPONDING AUTHOR:
}

Name: Ben L Zarzaur, MD MPH

Address: 702 Rotary Circle; Indianapolis, IN 46202

Telephone: (317) 962-5317

Fax: (317) 274-7426

Current E-mail: bzarzaur@iupui.edu

REPRINTS: Reprints will not be available from the authors.

DISCLAIMERS: Dr.'s Dolejs. Savage, Hartwell, and Zarzaur have no conflicts of interest to disclose.

FUNDING: There is no funding for this paper

MEETING: This was a podium presentation at the American Association for the Surgery of Trauma on September 14-17, 2016 in Waikoloa, HI

WORD COUNT TEXT: 3,492/3,500

WORD COUNT ABSTRACT: 247/250

REFERENCES: 37/50

Figure/Table Limit: 6/7

RUNNING TITLE: Trends in splenectomy over time 


\section{INTRODUCTION:}

Non-operative management (NOM) for blunt splenic injury (BSI) began over 30 years ago, and selective NOM is now the preferred method of treatment in BSI ${ }^{1-4}$. The use of angiography and embolization (ANGIO) in high-grade BSI began in 1979 and published in 1995 by Sclafani et al $^{2}$. Trauma centers then developed protocols incorporating the use of ANGIO in the management of BSI. Some centers follow selective ANGIO strategies where patients with BSI and a vascular abnormality seen on admission computed tomography (CT) scan are referred for ANGIO ${ }^{5-8}$. Other centers, based on evidence that CT may not be accurate at detecting vascular abnormalities that require embolization, advocate for the use of ANGIO for all patients with grades III-V or IV-V BSI, depending on the center ${ }^{9-16}$. Utilizing non-selective ANGIO strategies, authors report improvements in the proportion of patients successfully managed with NOM after protocol implementation. However, not all studies show improved splenic salvage with ANGIO ${ }^{17-20}$. A recent multicenter prospective study showed no association between ANGIO utilization and splenic salvage in patients selected for NOM ${ }^{17}$.

To further complicate matters, the use of angiography and embolization was introduced at a time when CT scanning in trauma was rapidly expanding and the rate of successful NOM of BSI was increasing across institutions, with or without ANGIO 14,21-23. An increase in diagnosis of relatively minor spleen injuries could lead to confounding of the relationship between ANGIO and splenic salvage ${ }^{14,21-23}$. Additionally, selection bias may also confound the relationship between ANGIO and splenic salvage. Many studies of ANGIO in the setting of BSI only report success of non-operative management ${ }^{5-15}$. Over time, changes in the use of urgent, or early splenectomy at a center could account for improvement in non-operative success. For example, if a center more accurately predicts which patients with BSI will ultimately fail observation and 
require splenectomy, the surgeons at that center may become more aggressive with the use of early splenectomy and not refer patients to ANGIO. If this occurs, the population referred to ANGIO will be enriched with patients more likely to have successful splenic salvage, as the highest risk patients have already undergone splenectomy. Thus, rates of NOM will improve compared to historical controls, not because of ANGIO but because of better patient selection. A recent study using the National Trauma Data Bank found significant variability in the use of urgent splenectomy at the center level indicating that there may be selection bias at the center level ${ }^{24}$.

In this study we sought to exploit center level heterogeneity in the utilization of ANGIO to investigate nationwide trends in ANGIO and splenectomy in centers that utilized ANGIO compared to centers that did not utilize ANGIO from 2008 to 2014. We hypothesized that the overall rate of splenectomy would be stable from 2008 to 2014 with an increased rate of early splenectomy and decreased rate of late splenectomy. We further hypothesized that these trends would be similar in centers that utilized ANGIO and those that did not.

\section{METHODS:}

Study Sample:

The American College of Surgeons (ACS) National Trauma Data Bank (NTDB) from 2008-2014 was used for this study. Adults 18 years and older with a BSI and abbreviated injury scale (AIS) over II (classified as high-grade BSI in our study) treated at Level I or II trauma centers were included. Patients were only included if they were treated at trauma centers that managed over 20 patients with high-grade BSI during the study period. Patients were excluded if 
they were dead on arrival, died in the emergency department (ED), or if they were transferred from another facility.

\section{Study Variables:}

Patient and facility level factors were examined. Patient characteristics included age, gender, race, admission systolic blood pressure (SBP), injury severity score (ISS), splenic injury severity (based on AIS), and the initial Glasgow coma scale (GCS) motor score with a priori defined cutoffs for continuous variables. Facilities were grouped based on ACS verification status, trauma level status, hospital region, number of beds, university status, and the usage of angiography at the facility level at any time during the study period.

Patients were classified as undergoing splenectomy if they had an International Classification of Diseases, Ninth Revision (ICD-9) procedure code of 41.5. Early splenectomy was defined as splenectomy within 6 hours of admission. Late splenectomy was defined as splenectomy over 6 hours from admission. ANGIO use was defined based on ICD-9 procedure codes of 88.47 (angiography) or 39.79 (angiography and embolization).

\section{Statistical Methods:}

Multiple imputation was performed for covariates with missing data. This included imputation for gender (0.2\% missing), GCS motor score (2.4\% missing), hospital region (0.4\% missing), and hour to splenectomy (5.5\% missing). Univariate and bivariate data analysis was then undertaken to compare unadjusted statistics over time. Statistical inference was performed with Chi-squared tests. 
We then used the multiply imputed dataset to perform hierarchal logistic regression analysis, clustered on facilities, to adjust for patient and facility factors. The events of interest were splenectomy, angiography, and mortality. Using methods similar to those used by Abdelsattar et al and Cohen et al, expected event rates were determined for each outcome ${ }^{25,26}$. Expected events rates were determined by the hierarchal logistic regression based on patient level (age, gender, race, admission SBP, ISS, splenic injury severity, GCS motor score) and facility level information (ACS verification, trauma level, region, number of beds, and university hospital status). Observed to expected (O:E) event ratios were then calculated for each year stratified by whether a center utilized ANGIO or not. Adjusted event rates for each year were then obtained by multiplying the O:E ratio and the overall event rate.

Next the O:E ratios were used to calculate a rate ratio (RR) for year of admission for ANGIO and non-ANGIO centers, with year considered as a linear, continuous variable, via a Poisson regression analysis. A rate ratio of 0.8 would mean that there was an average of a $20 \%$ reduction in the rate for each year of the study period. Interaction between year and ANGIO centers was ascertained for all estimates.

To investigate the role late splenectomy had on mortality, we utilized a Cox-proportional hazard model with late splenectomy as a time-varying covariate. Patients who had undergone early splenectomy or who died within 6 hours of admission (prior to the time they would be eligible for splenectomy) were excluded from this analysis. This analysis was adjusted for the same patient and facility level covariates described above.

All analyses were performed with SAS version 9.4 (Cary, NC), and all figures were created using R version 3.3.2. This study was exempt from Institutional Review Board approval at Indiana University. 


\section{RESULTS:}

In total, 53,689 patients with Grade III or higher BSI were included for analysis with nearly even distribution across each year. Considering the entire cohort, 16,375 patients (30.5\%) had a Grade III BSI, 28,079 patients (52.3\%) had a Grade IV BSI, and 9,235 patients (17.2\%) had a Grade V BSI. Patients treated at ANGIO centers had higher ISS, more commonly had Grade IV BSI, and had lower admission GCS motor scores (Table 1). ANGIO centers were more commonly Level I centers, were more commonly university affiliated, and tended to be larger (Table 1). All patient and facility level information shown in Table 1 were included in our adjusted models.

Angiography Rate:

In centers that utilized ANGIO, the rate of ANGIO steadily and significantly increased from 2008 to 2014 across all severities (Figure 1). For all high-grade BSI, the adjusted rate of ANGIO increased by 16\% per year (RR, 1.16 [95\% confidence interval (CI): 1.15-1.18]). ANGIO utilization also increased in grade III BSI (RR, 1.14 [95\% CI: 1.10-1.18]), grade IV BSI (RR, 1.17 [95\% CI: 1.15-1.20]), and grade V BSI (RR, 1.14 [95\% CI: 1.10-1.18]) when considered separately. If using 2008 as a reference group, the rate of ANGIO utilization increased from 5.7\% in 2008 to $14.1 \%$ in 2014 (difference, 8.4\% [95\% CI: 7.2\% to 9.7\%]).

\section{Overall Splenectomy Rate:}

The overall splenectomy rate has been stable over time across all splenic injury severities at ANGIO centers (Figure 2). In ANGIO centers, there was no change in the overall splenectomy 
rate amongst patients with grade III-V BSI considered jointly (RR, 1.00 [95\% CI: 0.99-1.00]) or grade III (RR, 0.99 [95\% CI: 0.96-1.01]), grade IV (RR, 1.00 [95\% CI: 0.98-1.01]), or grade V (RR, 1.00 [95\% CI: 0.99-1.01]) considered separately. In contrast, the rate of overall splenectomy decreased in patients treated at non-ANGIO centers with grade III-V BSI considered jointly (RR, 0.96 [95\% CI: 0.93-0.99]) and with grade III (RR, 0.84 [95\% CI: 0.760.92]) and grade IV BSI (RR, 0.95 [95\% CI: 0.90-0.99]) considered separately. The rate of overall splenectomy did not significantly change in patients treated at non-ANGIO centers with grade V BSI (RR, 1.01 [95\% CI: 0.96-1.06]). There was significant interaction between ANGIO center and year in all high-grade BSI (p-value=0.02), grade III BSI (p-value<0.01), and grade IV BSI (p-value=0.04) but not in grade V BSI (p-value=0.72). This indicates that the overall splenectomy rate decreased substantially more in non-ANGIO centers compared to ANGIO centers for all severities but grade V BSI, where there was no significant difference. In all highgrade BSI using 2008 as a reference, patients treated at an ANGIO center had a rate of overall splenectomy of 24.1\% in 2008 and 23.5\% in 2014 (difference, -0.7\% [95\% CI: -1.9\% to 0.6\%]) versus patients treated at a non-ANGIO center who had a rate of $27.0 \%$ in 2008 and $20.7 \%$ in 2014 (difference, $-6.3 \%$ [95\% CI: $-9.9 \%$ to $-2.0 \%]$ ).

\section{Early Splenectomy:}

The rate of early splenectomy was stable over time across all splenic injury severities in ANGIO and non-ANGIO centers, with the exception of grade III BSI treated at non-ANGIO centers (Figure 3). In ANGIO centers, there was no significant change in all high-grade BSI (RR, 1.01 [95\% CI: 1.00-1.02]), grade III BSI (RR, 1.00 [95\% CI: 0.97-1.03]), grade IV BSI (RR, 1.01 [95\% CI: 0.99-1.02]), or grade V BSI (RR, 1.01 [95\% CI: 0.99-1.03]). In non-ANGIO 
centers, there was significantly reduced early splenectomy rates in grade III BSI (RR, 0.83 [95\% CI: 0.74-0.94) but no significant differences in all high-grade BSI (RR, 0.98 [95\% CI: 0.941.01]), grade IV BSI (RR, 0.96 [95\% CI: 0.91-1.01]), or grade V BSI (RR, 1.03 [95\% CI: 0.981.09]). There was significant interaction between year and ANGIO center status for only grade III BSI (p-value<0.01). This indicates a significant reduction in the early splenectomy in patients with grade III BSI treated at non-ANGIO centers compared with ANGIO centers, but no significant differences in the rate of early splenectomy between ANGIO and non-ANGIO centers.

\section{Late Splenectomy:}

The rate of late splenectomy was significantly reduced over time in ANGIO and nonANGIO centers (Figure 4). In ANGIO centers, late splenectomy significantly decreased over time in all high-grade BSI (RR, 0.95 [95\% CI: 0.93-0.97]), grade III BSI (RR, 0.94 [95\% CI: 0.89-0.99]), grade IV BSI (RR, 0.97 [95\% CI: 0.94-1.00]), and grade V BSI (RR, 0.95 [95\% CI: 0.91-0.98]). In non-ANGIO centers, late splenectomy significantly decreased in high-grade BSI (RR, 0.89 [95\% CI: 0.83-0.95]), grade III BSI (RR, 0.84 [95\% CI: 0.71-1.00]), and grade V BSI (RR, 0.88 [95\% CI: 0.77-1.00]). In patients with grade IV BSI treated at non-ANGIO centers, the late splenectomy did not significantly decrease (RR, 0.91 [95\% CI: 0.82-1.00], p-value=0.06) despite a larger effect size than ANGIO centers. Interaction amongst ANGIO centers, nonANGIO centers, and year was nearly significant when all high-grade BSI was considered jointly (p-value=0.057), but was not significant in grade III BSI (p-value=0.23), grade IV BSI (pvalue $=0.21$ ), or grade V BSI (p-value=0.26). This indicates a trend towards a faster decline in the rate of late splenectomy in non-ANGIO centers compared to ANGIO centers in all high-grade 
BSI. Using 2008 as a reference year, the late splenectomy rate in high-grade BSI in patients treated at ANGIO centers decreased from 5.4\% in 2008 to 4.1\% in 2014 (difference, -1.4\% [95\% CI: $-1.8 \%$ to $-0.8 \%]$ ) and in patients treated at non-ANGIO centers decreased from $6.0 \%$ in 2008 to $3.3 \%$ in 2014 (difference, -3.5\% [95\% CI: $-4.6 \%$ to $-1.7 \%$ ]).

\section{Mortality:}

There were no significant changes in the rate of mortality over time for patients with high-grade BSI in ANGIO or non-ANGIO centers. In patients treated at ANGIO centers, there was no difference in mortality in high-grade BSI (RR, 1.01 [95\% CI: 0.99-1.02]), grade III BSI (RR, 1.01 [95\% CI: 0.98-1.05]), grade IV BSI (RR, 1.00 [95\% CI: 0.98-1.03]), or grade V BSI (RR, 1.01 [95\% CI: 0.99-1.03]). In patients treated at non-ANGIO centers, there was no difference in mortality in high-grade BSI (RR, 0.96 [95\% CI: 0.91-1.01]), grade III BSI (RR, 0.96 [0.84-1.08]), grade IV BSI (RR, 0.92 [95\% CI: 0.85-1.00]), or grade V BSI (RR, 0.99 [95\% CI: 0.80-1.04]). There was a trend towards interaction between ANGIO center and mortality in high-grade BSI ( $\mathrm{p}$-value=0.054) and grade IV BSI ( $\mathrm{p}$-value=0.054) but not in grade III BSI (pvalue $=0.39$ ) or grade V BSI (p-value $=0.58)$. Using 2008 as a reference year, the mortality in patients with high-grade BSI treated at ANGIO centers increased from 9.8\% in 2008 to $10.3 \%$ in 2014 (difference, $0.4 \%$ [95\% CI: $-0.4 \%$ to $1.3 \%$ ]) and at non-ANGIO centers decreased from $11.5 \%$ in 2008 to $6.6 \%$ in 2014 (difference, $-2.6 \%$ [95\% CI: $-4.9 \%$ to $0.4 \%$ ]).

We next evaluated mortality among patients with late splenectomy. Late splenectomy was associated with a significantly increased rate of mortality in grade III BSI (hazard ratio (HR), 1.98 [95\% CI: 1.32-2.96]) and grade IV BSI (HR, 1.69 [95\% CI: 1.35-2.10]). For Grades 
V BSI, there was no significant difference in mortality in patients who underwent late splenectomy (HR, 1.01 [95\% CI: 0.79, 1.29]).

\section{DISCUSSION:}

Utilizing the NTDB, we studied 53,689 patients who sustained high-grade BSI. Several retrospective studies indicate an association between ANGIO and decreased rates of failure of NOM $^{2,3,5-15,27-30}$. Further, several centers now advocate ANGIO for all Grade III to V or IV to V BSI ${ }^{9-15}$. Thus, the $8.4 \%$ increase rate of ANGIO from 2008 to 2014 is not surprising. It is also not surprising that the greatest change occurred amongst patients with Grade IV BSI, where the rate of ANGIO increased by 10.8\%. In this analysis, we found no change in the utilization of early splenectomy over time at ANGIO and non-ANGIO centers. This finding was contrary to our initial hypothesis and suggests that selection bias may not be a factor in the relationship between ANGIO and splenic salvage. However, we did find interesting results when considering overall and late splenectomy rates at ANGIO and non-ANGIO centers.

If increased ANGIO use on a nationwide basis resulted in improved splenic salvage, we would expect centers that utilized ANGIO frequently would have decreased overall and late splenectomy rates compared with centers that did not utilize ANGIO. However, our study shows that the overall splenectomy rate in centers that utilized ANGIO remained stable despite increasing ANGIO utilization rates. In contrast, non-ANGIO centers experienced significant decreases in the rate of overall splenectomy. Both ANGIO and non-ANGIO centers experienced decreased rates of late splenectomy across all severities. However, the rate of decrease tended to be steeper in non-ANGIO centers than in ANGIO centers. Regardless, this means that ANGIO centers and non-ANGIO centers experienced a similar change in late splenectomy over time. 
Thus, increased ANGIO utilization was not the only factor that drove decreased utilization of late splenectomy over time.

The reason for the decreased overall splenectomies in non-ANGIO centers and decreased late splenectomy in ANGIO and non-ANGIO centers is not entirely clear. In a large multiinstitutional study by Peitzman et al, only one-third of centers had a protocol for management of BSI, and studies have shown that implementation of a protocol (regardless of ANGIO utilization), results in improved late splenectomy rates ${ }^{1,9,11,31-33}$. It is possible that non-ANGIO centers and ANGIO centers both developed protocols that improved splenic salvage during the study period. Massive transfusion protocols were also developing and expanding during the study period ${ }^{34,35}$. It is possible that these protocols, or other unmeasured temporal trends, were associated with improvements in splenic salvage. Further, non-ANGIO centers were predominantly Level II, non-university affiliated, and small ( $<400$ beds) centers that treated on average 12.5 patients with high-grade BSI per center per year compared with 19.2 patients per center per year in ANGIO centers. It is possible that these centers were slower to adopt protocols for BSI, and after adoption, had heightened rate of splenic salvage compared with ANGIO centers. Regardless of the mechanism, our study provides evidence that increased ANGIO utilization is not the only driver of decreased late splenectomy rates over time in patients with high-grade BSI.

With increasing rates of ANGIO use, it is important to consider the complications associated with ANGIO. In a study of 88 patients who underwent ANGIO, ANGIO was associated with a $14 \%$ risk of major complications including splenic abscesses, infarction, cysts, and contrast-induced renal-insufficiency ${ }^{36}$. In another study, the morbidity of patients undergoing NOM with ANGIO was equivalent to the morbidity of patients undergoing 
immediate splenectomy ${ }^{18}$. Frandon et al. reported a $4 \%$ major complication rate amongst patients with BSI undergoing ANGIO with a 56\% minor complication rate ${ }^{37}$. In a multiinstitution study by Haan et al, the rate of major complication was $20 \%$ and minor complication was $23 \%{ }^{38}$. The rapid increase in ANGIO usage has significant cost implications. The procedure related charges for splenic embolization is estimated to be $\$ 19,062$ per embolization with similar per patient total hospital cost and total hospital charges ${ }^{39}$. Based on an estimate of 39,000 patients experiencing a BSI every year, the increase in ANGIO utilization we saw in our study resulted in an additional $\$ 194$ million dollars of healthcare costs from 2008 to $2014^{1,28,40}$. If we assume $20 \%$ of patients with BSI have grade IV or V injuries and a nationwide policy was instituted adopting ANGIO for all grade IV and V BSI, this would result in \$149 million dollars per year of healthcare costs, potentially unnecessary as demonstrated by splenic salvage rates at non-ANGIO centers ${ }^{12,21}$. Lastly, The results of this study also provide a cautionary tale for overaggressive use of NOM in BS since we observed a significant increase in mortality for patients with Grade III and Grade IV BSI who undergo splenectomy greater than 6 hours from admission.

This study does have important limitations. Residual confounding may have occurred based on year-by-year confounders that were not measured or for which an inappropriate adjustment was made. For example, institution of massive transfusion protocols, increased utilization of written protocols for the management of BSI, changes in trends in patient medication, such as increased use of direct thrombin inhibitors, or specific imaging characteristics such as arterial blush are not included in the NTDB and may confound our results. By comparing year-to-year trends and center level information, rather than patient level information, many of these possible unmeasured confounders are likely to be experienced 
similarly across institutions, which would minimize confounding in the relationship between ANGIO and non-ANGIO centers. Information bias is also of concern. If over time recording patterns at facilities changed systematically, these changes could significantly influence our results. The steady rate of splenectomy in ANGIO centers and the progressive and near linear increase in angiography makes it less likely that information bias was impacting our results. Further, facilities have a vested interest to accurately report angiography and splenectomy given the reimbursement associated with these codes.

Another potential bias is misclassification of splenic injury severity based on AIS, since radiologic information is not available from the NTDB. It is likely that over-time, individual facilities would report a similar AIS for the same pattern of BSI, so the year-by-year comparison is unlikely to be influenced by this effect. Another limitation was that the most frequent source of missing data was in the time to splenectomy (5.5\% missing). This time was imputed and patients were grouped as having early or late splenectomies based on an a priori defined cutoff of 6 hours. It is possible that imputation influenced the rate of early or late splenectomy. Imputation would not change the rate of overall splenectomy. Further, a sensitivity analysis performed with and without the imputed data revealed similar trends in the rate of early and late splenectomy.

In light of these limitations, our study demonstrates that despite the rapidly expanding use of ANGIO, the overall splenectomy rate remained stable for high-grade BSI in centers that utilized ANGIO but decreased in centers that did not utilize ANGIO. Late splenectomy rates decreased at similar rates in ANGIO and non-ANGIO centers and, may be decreasing more rapidly in non-ANGIO centers. At an institutional level, increased ANGIO utilization does not appear to impact the rates of overall splenectomy or late splenectomy. The role of ANGIO in 
BSI needs to be better clarified by a large, multi-institutional randomized control trial in order to provide the information needed to make informed decisions about ANGIO in the setting of $\mathrm{BSI}^{17}$. Until such a trial is undertaken, on a population basis, expanding the use of ANGIO does not appear to impact the overall rate of splenic salvage. 
TABLES AND FIGURES: 
Table 1: Patient and hospital characteristics in ANGIO and non-ANGIO centers. ${ }^{*} \mathrm{SBP}=$ systolic blood pressure, GCS=Glasgow coma score, ACS=American College of Surgeons, ANGIO=Splenic angiography or embolization

\begin{tabular}{|c|c|c|c|}
\hline Year & ANGIO Center & Non-ANGIO Center & p-value \\
\hline \multicolumn{4}{|l|}{ Patient Level Information: } \\
\hline Number of Patients: & 49,401 & 4,288 & \\
\hline \multicolumn{4}{|l|}{ Year, \% } \\
\hline 2008 & 13.8 & 13.3 & \multirow{7}{*}{0.37} \\
\hline 2009 & 12.9 & 13.3 & \\
\hline 2010 & 13.3 & 14.4 & \\
\hline 2011 & 14.2 & 13.8 & \\
\hline 2012 & 16.0 & 15.3 & \\
\hline 2013 & 14.9 & 14.8 & \\
\hline 2014 & 15.0 & 15.1 & \\
\hline Age $\geq 55 \mathrm{y}, \%$ & 20.6 & 20.3 & 0.56 \\
\hline Male, \% & 67.9 & 67.3 & 0.42 \\
\hline Non-white, \% & 24.9 & 24.4 & 0.47 \\
\hline Admission SBP<90 mmHg, \% & 13.4 & 13.6 & 0.73 \\
\hline \multicolumn{4}{|l|}{ Injury Severity Score, \% } \\
\hline $0-10$ & 10.1 & 14.7 & \multirow{3}{*}{$<0.01$} \\
\hline $10-24$ & 43.8 & 43.3 & \\
\hline$\geq 25$ & 46.1 & 42.1 & \\
\hline \multicolumn{4}{|l|}{ Splenic Injury Severity, \% } \\
\hline 3 & 30.3 & 32.8 & \multirow{3}{*}{$<0.01$} \\
\hline 4 & 52.6 & 48.8 & \\
\hline 5 & 17.1 & 18.4 & \\
\hline \multicolumn{4}{|l|}{ GCS Motor Score, \% } \\
\hline 6 & 74.1 & 76.1 & \multirow{3}{*}{$<0.01$} \\
\hline $2-5$ & 8.3 & 9.7 & \\
\hline 1 & 17.6 & 14.2 & \\
\hline \multicolumn{4}{|l|}{ Facility Level Information: } \\
\hline Number of Facilities: & 367 & 49 & \\
\hline ACS Certified, \% & 59.1 & 66.2 & 0.26 \\
\hline Level I Center, \% & 49.9 & 17.8 & $<0.01$ \\
\hline \multicolumn{4}{|l|}{ Region, \% } \\
\hline Northeast & 27.1 & 35.6 & \multirow{4}{*}{0.25} \\
\hline Midwest & 20.3 & 12.3 & \\
\hline South & 31.2 & 27.4 & \\
\hline West & 21.4 & 24.7 & \\
\hline \multicolumn{4}{|l|}{ Bed size, \% } \\
\hline$\leq 200$ & 5.2 & 12.2 & \multirow{4}{*}{$<0.01$} \\
\hline $201-400$ & 31.1 & 50.0 & \\
\hline $401-600$ & 33.2 & 25.7 & \\
\hline$>600$ & 30.5 & 12.2 & \\
\hline University Affiliated, \% & 43.1 & 21.6 & $<0.01$ \\
\hline
\end{tabular}




\section{FIGURE LEGENDS}

Figure 1: Adjusted rates of angiography over time. The line for angiography centers and nonangiography centers represents a fitted line obtained from a Poisson regression. Rates were adjusted for patient level (age, gender, race, admission SBP, ISS, splenic injury severity, GCS motor score) and facility level information (ACS verification, trauma level, region, number of beds, and university hospital status).

Figure 2: Adjusted rates of overall splenectomy over time. The line for angiography centers and non-angiography centers represents a fitted line obtained from a Poisson regression. Rates were adjusted for patient level (age, gender, race, admission SBP, ISS, splenic injury severity, GCS motor score) and facility level information (ACS verification, trauma level, region, number of beds, and university hospital status).

Figure 3: Adjusted rates of early splenectomy over time. The line for angiography centers and non-angiography centers represents a fitted line obtained from a Poisson regression. Rates were adjusted for patient level (age, gender, race, admission SBP, ISS, splenic injury severity, GCS motor score) and facility level information (ACS verification, trauma level, region, number of beds, and university hospital status).

Figure 4: Adjusted rates of late splenectomy over time. The line for angiography centers and non-angiography centers represents a fitted line obtained from a Poisson regression. Rates were adjusted for patient level (age, gender, race, admission SBP, ISS, splenic injury severity, GCS motor score) and facility level information (ACS verification, trauma level, region, number of beds, and university hospital status).

Figure 5: Adjusted rates of mortality over time. The line for angiography centers and nonangiography centers represents a fitted line obtained from a Poisson regression. Rates were adjusted for patient level (age, gender, race, admission SBP, ISS, splenic injury severity, GCS motor score) and facility level information (ACS verification, trauma level, region, number of beds, and university hospital status). 


\section{CITATIONS:}

1. Peitzman AB, Heil B, Rivera L, et al. Blunt splenic injury in adults: Multi-institutional Study of the Eastern Association for the Surgery of Trauma. J Trauma. 2000;49(2):177-187; discussion 187179.

2. Sclafani SJ, Shaftan GW, Scalea TM, et al. Nonoperative salvage of computed tomographydiagnosed splenic injuries: utilization of angiography for triage and embolization for hemostasis. Journal of Trauma-Injury Infection \& Critical Care. 1995;39(5):818-825; discussion 826-817.

3. Scarborough JE, Ingraham AM, Liepert AE, Jung HS, O'Rourke AP, Agarwal SK. Non-Operative Management Is As Effective As Immediate Splenectomy For Adult Patients with High-Grade Blunt Splenic Injury. J Am Coll Surg. 2016.

4. Stassen NA, Bhullar I, Cheng JD, et al. Selective nonoperative management of blunt splenic injury: an Eastern Association for the Surgery of Trauma practice management guideline. The journal of trauma and acute care surgery. 2012;73(5 Suppl 4):S294-300.

5. Watson GA, Hoffman MK, Peitzman AB. Nonoperative management of blunt splenic injury: what is new? Eur J Trauma Emerg Surg. 2015;41(3):219-228.

6. Olthof $\mathrm{DC}$, van der Vlies $\mathrm{CH}$, Joosse $\mathrm{P}$, et al. Consensus strategies for the nonoperative management of patients with blunt splenic injury: a Delphi study.[Erratum appears in J Trauma Acute Care Surg. 2013 Aug;75(2):353-4 Note: van der Vlies, Cornelius H [corrected to van der Vlies, Cornelis H]]. The journal of trauma and acute care surgery. 2013;74(6):1567-1574.

7. van der Vlies CH, Hoekstra J, Ponsen KJ, Reekers JA, van Delden OM, Goslings JC. Impact of splenic artery embolization on the success rate of nonoperative management for blunt splenic injury. Cardiovasc Intervent Radiol. 2012;35(1):76-81.

8. Ekeh AP, Izu B, Ryan M, McCarthy MC. The impact of splenic artery embolization on the management of splenic trauma: an 8-year review. Am J Surg. 2009;197(3):337-341.

9. Skattum J, Naess PA, Eken T, Gaarder C. Refining the role of splenic angiographic embolization in high-grade splenic injuries. The journal of trauma and acute care surgery. 2013;74(1):100-103; discussion 103-104.

10. Dehli T, Bagenholm A, Trasti NC, Monsen SA, Bartnes K. The treatment of spleen injuries: a retrospective study. Scand J Trauma Resusc Emerg Med. 2015;23:85.

11. Miller PR, Chang MC, Hoth JJ, et al. Prospective trial of angiography and embolization for all grade III to $\mathrm{V}$ blunt splenic injuries: nonoperative management success rate is significantly improved. J Am Coll Surg. 2014;218(4):644-648.

12. Bhullar IS, Frykberg ER, Tepas JJ, 3rd, Siragusa D, Loper T, Kerwin AJ. At first blush: absence of computed tomography contrast extravasation in Grade IV or V adult blunt splenic trauma should not preclude angioembolization. The journal of trauma and acute care surgery. 2013;74(1):105111; discussion 111-102.

13. Bhullar IS, Frykberg ER, Siragusa D, et al. Selective angiographic embolization of blunt splenic traumatic injuries in adults decreases failure rate of nonoperative management. The journal of trauma and acute care surgery. 2012;72(5):1127-1134.

14. Sabe AA, Claridge JA, Rosenblum DI, Lie K, Malangoni MA. The effects of splenic artery embolization on nonoperative management of blunt splenic injury: a 16-year experience. $J$ Trauma. 2009;67(3):565-572; discussion 571-562.

15. Gaarder C, Dormagen JB, Eken T, et al. Nonoperative management of splenic injuries: improved results with angioembolization. J Trauma. 2006;61(1):192-198.

16. Marmery H, Shanmuganathan K, Mirvis SE, et al. Correlation of multidetector CT findings with splenic arteriography and surgery: prospective study in 392 patients. J Am Coll Surg. 2008;206(4):685-693. 
17. Zarzaur BL, Kozar R, Myers JG, et al. The splenic injury outcomes trial: An American Association for the Surgery of Trauma multi-institutional study. The journal of trauma and acute care surgery. 2015;79(3):335-342.

18. Chastang L, Bege $\mathrm{T}$, Prudhomme $\mathrm{M}$, et al. Is non-operative management of severe blunt splenic injury safer than embolization or surgery? Results from a French prospective multicenter study. $J$ Visc Surg. 2015;152(2):85-91.

19. Harbrecht BG, Ko SH, Watson GA, Forsythe RM, Rosengart MR, Peitzman AB. Angiography for blunt splenic trauma does not improve the success rate of nonoperative management. $J$ Trauma. 2007;63(1):44-49.

20. Duchesne JC, Simmons JD, Schmieg RE, Jr., McSwain NE, Jr., Bellows CF. Proximal splenic angioembolization does not improve outcomes in treating blunt splenic injuries compared with splenectomy: a cohort analysis. J Trauma. 2008;65(6):1346-1351; discussion 1351-1343.

21. Harbrecht BG, Zenati MS, Ochoa JB, Puyana JC, Alarcon LH, Peitzman AB. Evaluation of a 15-year experience with splenic injuries in a state trauma system. Surgery. 2007;141(2):229-238.

22. Clancy AA, Tiruta C, Ashman D, Ball CG, Kirkpatrick AW. The song remains the same although the instruments are changing: complications following selective non-operative management of blunt spleen trauma: a retrospective review of patients at a level I trauma centre from 1996 to 2007. Journal of trauma management \& outcomes. 2012;6(1):4.

23. Soo KM, Lin TY, Chen CW, et al. More becomes less: management strategy has definitely changed over the past decade of splenic injury--a nationwide population-based study. Biomed Res Int. 2015;2015:124969.

24. Zarzaur BL, Croce MA, Fabian TC. Variation in the use of urgent splenectomy after blunt splenic injury in adults. J Trauma. 2011;71(5):1333-1339.

25. Abdelsattar ZM, Krell RW, Campbell DA, Jr., Hendren S, Wong SL. Differences in hospital performance for noncancer vs cancer colorectal surgery. J Am Coll Surg. 2014;219(3):450-459.

26. Cohen ME, Dimick JB, Bilimoria KY, Ko CY, Richards K, Hall BL. Risk adjustment in the American College of Surgeons National Surgical Quality Improvement Program: a comparison of logistic versus hierarchical modeling. J Am Coll Surg. 2009;209(6):687-693.

27. Olthof DC, Joosse P, van der Vlies CH, de Haan RJ, Goslings JC. Prognostic factors for failure of nonoperative management in adults with blunt splenic injury: a systematic review. The journal of trauma and acute care surgery. 2013;74(2):546-557.

28. Zarzaur BL, Savage SA, Croce MA, Fabian TC. Trauma center angiography use in high-grade blunt splenic injuries: Timing is everything. The journal of trauma and acute care surgery. 2014;77(5):666-673.

29. Wei B, Hemmila MR, Arbabi S, Taheri PA, Wahl WL. Angioembolization reduces operative intervention for blunt splenic injury. J Trauma. 2008;64(6):1472-1477.

30. van der Vlies CH, van Delden OM, Punt BJ, Ponsen KJ, Reekers JA, Goslings JC. Literature review of the role of ultrasound, computed tomography, and transcatheter arterial embolization for the treatment of traumatic splenic injuries. Cardiovasc Intervent Radiol. 2010;33(6):1079-1087.

31. Starnes S, Klein P, Magagna L, Pomerantz R. Computed tomographic grading is useful in the selection of patients for nonoperative management of blunt injury to the spleen. Am Surg. 1998;64(8):743-748; discussion 748-749.

32. Claridge JA, Carter JW, McCoy AM, Malangoni MA. In-house direct supervision by an attending is associated with differences in the care of patients with a blunt splenic injury. Surgery. 2011;150(4):718-726.

33. Peitzman AB, Harbrecht BG, Rivera L, Heil B, Eastern Association for the Surgery of Trauma Multiinstitutional Trials W. Failure of observation of blunt splenic injury in adults: variability in practice and adverse consequences. J Am Coll Surg. 2005;201(2):179-187. 
34. Cotton BA, Gunter OL, Isbell J, et al. Damage control hematology: the impact of a trauma exsanguination protocol on survival and blood product utilization. J Trauma. 2008;64(5):11771182; discussion 1182-1173.

35. O'Keeffe T, Refaai M, Tchorz K, Forestner JE, Sarode R. A massive transfusion protocol to decrease blood component use and costs. Arch Surg. 2008;143(7):686-690; discussion 690-681.

36. Ekeh AP, Khalaf S, Ilyas S, Kauffman S, Walusimbi M, McCarthy MC. Complications arising from splenic artery embolization: a review of an 11-year experience. Am J Surg. 2013;205(3):250-254; discussion 254.

37. Frandon J, Rodiere $\mathrm{M}$, Arvieux $\mathrm{C}$, et al. Blunt splenic injury: outcomes of proximal versus distal and combined splenic artery embolization. Diagnostic and interventional imaging. 2014;95(9):825-831.

38. Haan JM, Biffl W, Knudson MM, et al. Splenic embolization revisited: a multicenter review. Journal of Trauma-Injury Infection \& Critical Care. 2004;56(3):542-547.

39. Bruce PJ, Helmer SD, Harrison PB, Sirico T, Haan JM. Nonsurgical management of blunt splenic injury: is it cost effective? Am J Surg. 2011;202(6):810-815; discussion 815-816.

40. WISQARS Centers for Disease Control: National Center For Injury Prevention and Control; 2008. http://www.cdc.gov/ncipc/wisqars/. Accessed August 1, 2008. 
Adjusted Rate of Overall Splenectomy Over Time

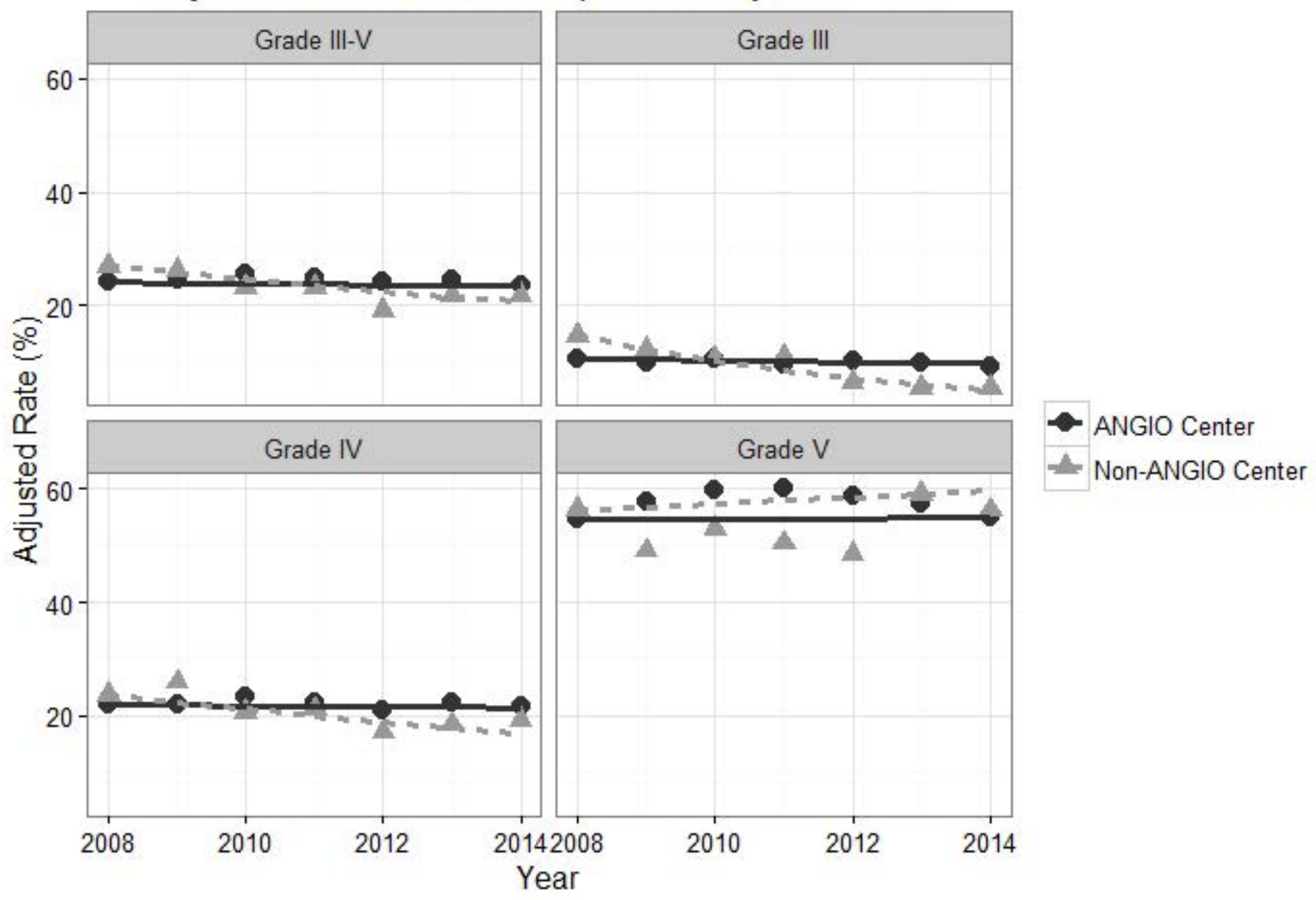


Adjusted Rate of Early Splenectomy Over Time

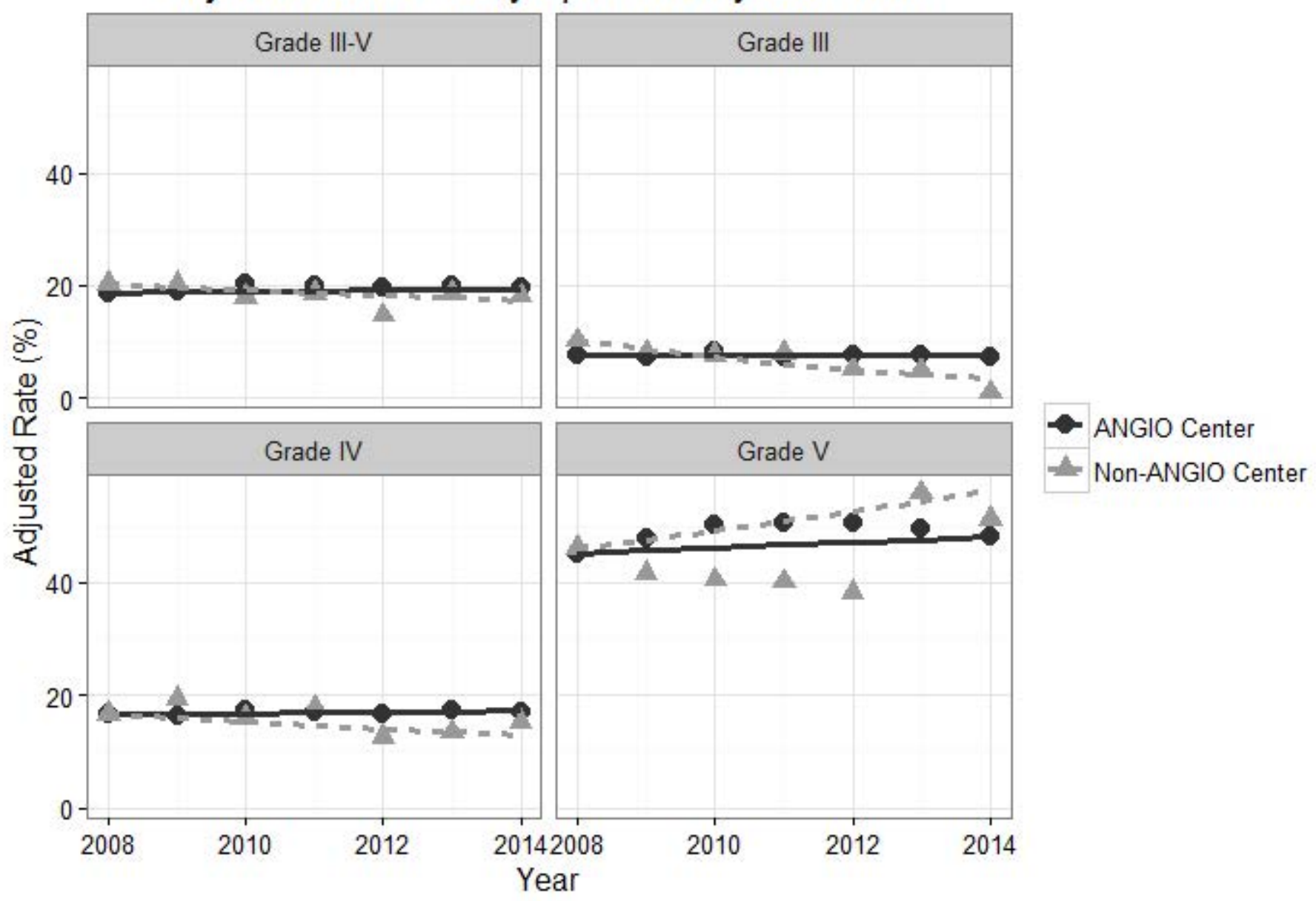


Adjusted Rate of Late Splenectomy Over Time

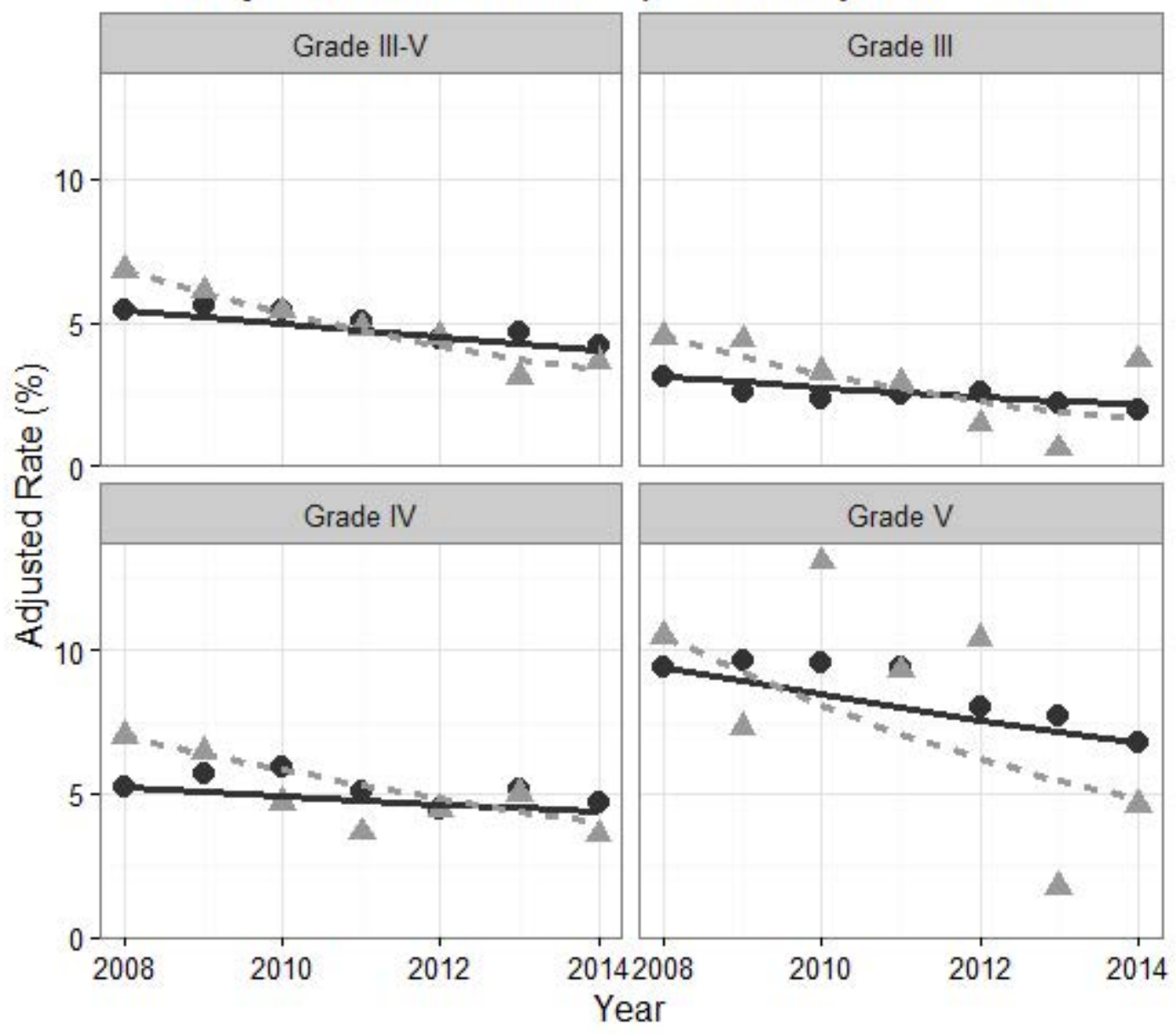

ANGIO Center Non-ANGIO Center 


\section{Adjusted Rate of Mortality Over Time}

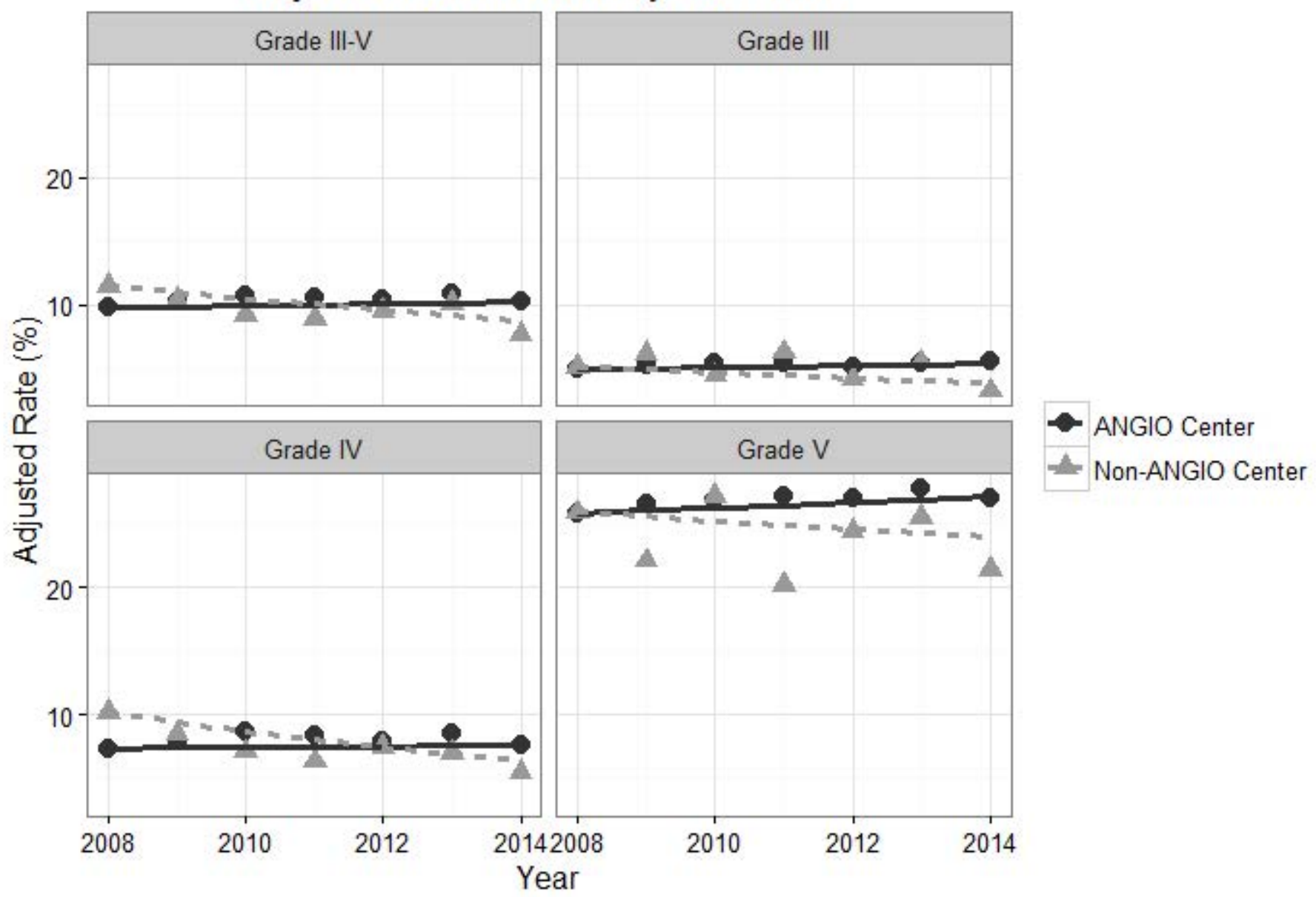

